\title{
Reinfection or Relapse in SARS-CoV-2-Infected Patients; Does it Occur?
}

\author{
Abdollah Karimi (iD) ${ }^{1}$, Fariba Shirvani ${ }^{1{ }^{*}}$ and Kimia Seifi ${ }^{2}$ \\ ${ }^{1}$ Pediatric Infections Research Center, Research Institute for Children's Health, Shahid Beheshti University of Medical Sciences, Tehran, Iran \\ ${ }^{2}$ Young Researchers and Elite Club, Roudehen Branch, Islamic Azad University, Roudehen, Iran \\ "Corresponding author: Pediatric Infections Research Center, Research Institute for Children Health, Shahid Beheshti University of Medical Sciences, Tehran, Iran. Email: \\ shirvanifariba@rocketmail.com
}

Received 2020 April 06; Revised 2020 April 22; Accepted 2020 April 23.

Keywords: Relapse, Reinfection, SARS-COV-2

\section{Dear Editor,}

The first pneumonia cases of unknown origin were found in Wuhan City, Hubei Province, China, in early December 2019. Using high-throughput sequencing, a novel coronavirus was detected in the throat swab sample of a patient on January 7. It was named as SARS-CoV-2 on February 11, 2020, by the International Committee on Taxonomy of Viruses (ICTV) after first being known as 2019-nCoV (1). The World Health Organization (WHO) announced COVID19 to be a "public health emergency of international concern" on January 30, 2020. This virus has a genetic similarity with SARS-CoV responsible for an epidemic in China in 2003 and MERS-CoV in Saudi Arabia in 2012 (2). There were 5939234 confirmed cases and 367225 deaths due to virus in 216 countries up to 30 May 2020 (3). The disease has various forms from asymptomatic to severe cases with acute respiratory distress syndrome, leading to death. The transmission of SARS-CoV-2 may occur in different stages of disease, even from asymptomatic cases or within the incubation period. Transmission occurs from person to person by aerosols, contact with fresh secretions, fecal-oral route, and perhaps airborne droplets.

The duration of viral shedding can range widely, possibly depending on illness severity. In a mild illness, it is about 10 days after the onset of symptoms, but in severe cases, it is reported up to 37 days (4). Important diagnostic tests are nasopharynx, oropharynx, bronchoalveolar lavage, stool RT-PCR, and chest CT scan. The sensitivity of RT-PCR alone is $78.2 \%$, which increases to $91.9 \%$ in combination with chest CT scan (1). Based on CDC recommendations, the discontinuation of home isolation is indicated using a test-based strategy, as follows: fever resolution without fever-reducing medications AND respiratory symptoms (such as cough, shortness of breath) improvement AND at least two negative results of consecutive nasopharyngeal swab specimens using an FDA Emergency Use Authorized molecular assay for COVID-19 $\geq 24$ hours apart (total of two negative specimens) (4). However, there have been patients whose negative tests became positive and remained positive for a long time.

Four medical personnel with COVID-19 infection at Zhongnan Hospital of Wuhan University, Wuhan, China, from January 1, 2020, to February 15, 2020, were quarantined at home. After recovery, they fulfilled the WHO criteria plus no abnormality in X-ray for return to work. Five to 13 days after the discontinuation of home quarantine, they underwent repeated RT-PCR tests, and all showed positivity; besides, three repeated tests were positive in the next four to five days. Using a different kit did not change the positivity of results. The patients were asymptomatic and did not develop new lesions on chest CT scan. An increasing number of discharged coronavirus patients in China and elsewhere became positive after recovery, which sometimes occurred weeks after hospital discharge. It is not clear whether patients in their convalescent period have a risk of "reinfection" or "relapse" (5). Thus, the issues of prolonged immunity, the possibility of reinfection, and disease control are discussed, and once the pandemic is over, it may demonstrate its value in assessing the image of the disease.

In a longitudinal tracking study of infected monkeys with SARS-CoV-2, the primary infection caused viral replication in different organs. After primary infection, half of the monkeys were rechallenged with the SARS-CoV-2 strain. Five days after rechallenge, no viral load was found in anal and nasopharyngeal swabs, and no viral replication was detected in all primary tissue compartments (6). The mechanisms possibly responsible for reinfection or relapse are as follows:

1) Like Enterovirus, Coronavirus may have a "biphasic" 
nature, at least in a few infected individuals. Thus, it can have a dormant phase before symptom reappearance.

2) Patients in the convalescent phase may not produce enough antibodies and remain sensitive to reinfection (7).

3) Reinfection and relapse may occur in patients receiving corticosteroids or those who are immunocompromised (4).

4) In cases where the test is initially positive, then becomes negative, and again positive, it may be due to wrong sampling, false kit, or kit change; thus, a negative case is wrongly negative; that is, the negativity is false.

5) The virus may have acquired the power to hide from the immune system and escaped from defensive cells for a short period (8).

6) Reinfection may occur in the evolution between the body, the virus, and environmental conditions.

7) Positive tests after recovery may also be due to residual viral RNA detection remaining in the body, but it is not high enough to cause disease. "Viral RNA can last a long time even after the actual virus has been stopped"' (9).

Whatever it is, now, it is not so much to affect the prevention strategy. In conclusion, we think it takes more time to prove the real reinfection with SARS-CoV-2, but if it is true, it makes the nature of the disease more dangerous. We need more research in this field and children's epidemiology.

\section{Footnotes}

Authors' Contribution: Abdollah Karimi, Fariba Shirvani, and Kimia Seifi designed the letter and its revision.
Ethical Approval: There is no need for ethical approval. Funding/Support: There is no funding support.

\section{References}

1. Ren X, Liu Y, Chen H, Liu W, Guo Z, Zhang Y, et al. Application and optimization of RT-PCR in diagnosis of SARS-CoV-2 infection. SSRNElectron J. 2020. doi: 10.2139/ssrn.3546086.

2. Wu D, Wu T, Liu Q, Yang Z. The SARS-CoV-2 outbreak: What we know. Int J Infect Dis. 2020;94:44-8. doi: 10.1016/j.ijid.2020.03.004. [PubMed: 32171952]. [PubMed Central: PMC7102543].

3. COVID-19 coronavirus pandemic,World meter. 2020. Available from: https://www.worldometers.info/coronavirus/\#countries.

4. McIntosh KM, Bloom A. Uptodate Literature review current through 2020, [cited 2020 Mar 27]. Available from: https://www.uptodate. com/contents/coronavirus-disease-2019-covid-19/print.

5. Lan L, Xu D, Ye G, Xia C, Wang S, Li Y, et al. Positive RT-PCR test results in patients recovered from COVID-19. JAMA. 2020. doi: 10.1001/jama.2020.2783. [PubMed: 32105304]. [PubMed Central: PMC7047852].

6. Bao L, Deng W, Gao H, Xiao C, Liu J, Xue J, et al. Reinfection could not occur in SARS-2 CoV-2 infected rhesus macaques. bioRxiv Preprint. 2020. doi: 10.1101/2020.03.13.990226.

7. Stanway D, Kelland K. Coronavirus reappears in discharged patients, raising questions in containment fight. World News; 2020, [cited 2020 Feb 28]. Available from: https://www.reuters.com/article/us-chinahealth-reinfection-explainer/explainer-coronavirus-reappearsin-discharged-patients-raising-questions-in-containment-fightidusken $20 \mathrm{~m} 124$.

8. Xie X, Zhong Z, Zhao W, Zheng C, Wang F, Liu J. Chest CT for typical 2019-nCoV Pneumonia: Relationship to negative RT-PCR testing. Radiology. 2020:200343. doi: 10.1148/radiol.2020200343. [PubMed: 32049601].

9. Leung H. Health COVID 19 Can You be re-infected after recovering from coronavirus? Here's what we know about COVID-19 immunity. Time; 2020, [cited 2020 Apr 03]. Available from: https://time.com/5810454/ coronavirus-immunity-reinfection/.

Conflict of Interests: There is no conflict of interest. 